\title{
PERFORMANCE EVALUATION OF AN INNOVATIVE INTEGRATED CHARGE-SENSITIVE PREAMPLIFIER WITH DYNAMIC RANGE BOOSTER
}

\author{
A. Pullia, S. Capra
}

\begin{abstract}
In this work we present an innovative all integrated solution, which incorporates on the same chip both a low-noise low-power charge-sensitive preamplifier and an integrated range booster with second-order effect compensation. The booster is based on a particular Time-to-Amplitude Converter which generates analog signals whose amplitude is directly proportional to the energy of the events that caused saturation (ions, energetic charged particles). In this way a dynamic range from a few $\mathrm{keV}$ to more than $800 \mathrm{MeV}$ is obtained.
\end{abstract}

\section{INTRODUCTION}

Integrated circuits suffer from the limited supply voltage they can tolerate without permanent damage. A reduced supply voltage yields a reduced output voltage swing. So, the overall dynamic range of integrated Charge-Sensitive Preamplifiers (CSP) is typically remarkably smaller than that obtained with discrete component solutions [1-6]. Losing the ability to measure the event energy is not the only problem associated with CSP saturation. Saturation translates into dead-time, i.e a certain window of time over which the preamplifier is not able to amplify/analyze the signals coming from the detector. We propose here an original circuit solution conceived to address these issues. Not only is the shown device able to reduce substantially the dead-time. It even allows high-resolution spectroscopy when the preamplifier is in deep saturation condition. For energies under the threshold of saturation the preamplifier works in a standard way, while for over-threshold events an auxiliary signal is generated the amplitude of which is proportional to the energy released into the detector.

\section{CONCEPT AND FUNCTIONALITY}

The energy measurement of over-threshold signals is based on a "fast-reset" circuitry [7]. A comparator recognizes when the output voltage of the CSP reaches the saturation point. It triggers a switched current sink which starts draining the excess charge away from the input node of the preamplifier, i.e. the output node of the detector, until the preamplifier output voltage is brought back to the $0 \mathrm{~V}$ baseline level. In this way a substantial reduction of the dead time is obtained from some milliseconds to some microseconds typically, with a

Manuscript received on December 5, 2015.

A. Pullia is with the Department of Physics, University of Milano, Italy, and INFN of Milano, Italy (email: alberto.pullia@mi.infn.it).

S. Capra is with the Physics Department of University of Milano and INFN of Milano, Italy (email: stefano.capra@unimi.it) dramatic benefit for the experiments. When a large event saturates the CSP, the charge generated by the detector is not lost. It is conserved on the capacitance of the input node [8]. We discharge the input node with a constant and controlled current sink. In this way we can deduce the amount of charge looking at the time taken for removing, at constant rate, the charge itself. A time-over-threshold analysis [9] is then performed on the comparator signal, whose duration is proportional to the removed charge. The effectiveness of this method has been demonstrated. However the method has a weak point. When performing a fast-reset cycle the residual charge of the tail of previous events is undistinguishable from the charge released by the detector in correspondence to the event we are measuring. The error due the presence of this stochastic charge could spoil the spectroscopic performances of the device, especially at high event rates. We hence designed a particular Time-to-Amplitude Converter (TAC) which not only converts the duration of the reset cycle into amplitude, but also corrects this one from the error induced by the tail observed at the beginning of the reset cycle. In this way the amplitude of the signals coming from the TAC is proportional to the energy of the last event only. The TAC signals can then be simply processed by a pulse-height analyzer. The TAC signals end with a nice flat-top which makes the sampling procedure easy. The height of these signals is proportional to the event energy up to $800 \mathrm{MeV}$. The original dynamic range of the preamplifier is thus extended by more than one order of magnitude. The circuit structure of the TAC is similar to that of a CAMEX filter. A multi-stage lowpass filter is used in combination with controlled switches to perform an average of the baseline of the CSP before and after the reset cycle. These two values are subtracted one from the other and then used to correct the amplitude of the integrator voltage inside the TAC. Since these pulses do not require any kind of shaping, the r.m.s. fluctuation of the TAC output voltage is proportional to its equivalent input noise. This procedure is triggered only for events with energy higher than $37 \mathrm{MeV}$. This means that the spectroscopic broadening introduced by noise is generally negligible. This should be good enough not only for particle [10] but also for gamma spectroscopy. This kind of circuit requires two power rails and thus cannot be used with probe-like preamplifiers [11]. 


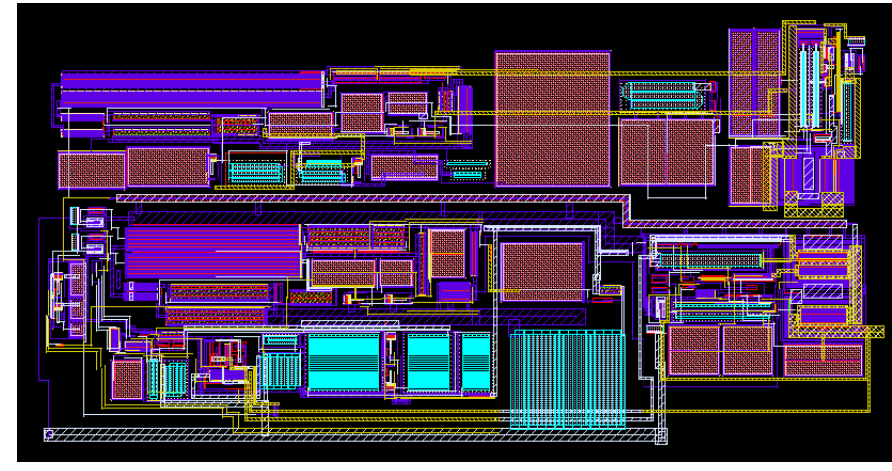

Fig. 1 - Layout of the CSP with the TAC structure. The device is 570 um x 1060um bonding pads excluded. It does not require any external component and both the CSP and the TAC are able to drive a $50 \Omega$ coaxial cable.

\section{CONCLUSIONS}

An innovative CSP was designed. It works in two different conditions for low-energy under-threshold signals and highenergy over-threshold ones. In the first case the energy resolution is determined by the CSP only. For events above the threshold of saturation the noise is generally not a concern. The auxiliary signals generated while working in the second conditions do not require shaping and can be sent directly to an ADC or analog memory [12-15]. The overall power consumption is $20 \mathrm{~mW}$ equally distributed between the preamplifier and the TAC device.

\section{REFERENCES}

[1] G. Pascovici et al., "Low noise, dual gain preamplifier with built in spectroscopic pulser for highly segmented high-purity germanium detectors", WSEAS Trans. on Circuits and Systems, vol. 7, no. 6, pp. 470-481, 2008.

[2] F. Zocca et al., "A Smart Reset Circuit for Low-Noise Preamplifiers of X$\gamma$ Ray Sensor Signals", IEEE Trans. Nucl. Sci., vol. 54, no. 1, pp. 197201, 2007.

[3] A. Pullia, G. Pascovici, C. Ur, "A versatile low-noise wide-range chargesensitive preamplifier for HPGe detectors", 2012 IEEE Nuclear Science Symposium and Medical Imaging Conf. Rec., ISBN 978-1-4673-2028-3, pp. 815-818, Anaheim, CA, Oct. 27 2012-Nov. 3 2012, DOI: 10.1109/NSSMIC.2012.6551217

[4] F. Zocca, A. Pullia, D. Bazzacco, and G. Pascovici, "A Time-OverThreshold Technique for Wide Dynamic Range Gamma-Ray Spectroscopy with the AGATA Detector", IEEE Trans. Nucl. Sci., vol. 56, no. 4, pp. 2384-2391, 2009

[5] A. Pullia et al., "An Advanced Preamplifier for Highly Segmented Germanium Detectors", IEEE Trans. Nucl. Sci., vol. 53, no. 5, pp. 28692875,2006
[6] A. Pullia et al., "Extending the dynamic range of nuclear pulse spectrometers", Rev. Sci. Instrum., vol. 79, p. 036105-01, 2008, doi: 10.1063/1.2894305

[7] S. Capra, D. Mengoni, R. J. Aliaga, A. Gadea, V. Gonzalez, A. Pullia, "Design of an Integrated Low-Noise, Low-Power Charge Sensitive Preamplifier for $\gamma$ and particle spectroscopy with solid state detectors", to be published in 2014 IEEE Nuclear Science Symposium and Medical Imaging Conference Record, NSS/MIC 2014, Seattle, WA

[8] Pullia, A., Capra, S., "Design of a resistorless ASIC preamplifier for HPGe detectors with non-linear pole/zero cancellation and controlled fast-reset feature", Published in 2012 IEEE Nuclear Science Symposium and Medical Imaging Conference Record, NSS/MIC 2012; Anaheim, CA; United States; 29 October 2012 through 3 November 2012; Category number CFP12NSS-ART; Code 98448. Article number 6551066, Pages 86-90 DOI: 10.1109 /NSSMIC.2012.6551066

[9] Pullia, A., Zocca, F., Capra, S., "An integrated low-noise charge-sensitive preamplifier with virtually unlimited spectroscopic dynamic range", Published in 2012 IEEE Nuclear Science Symposium and Medical Imaging Conference Record, NSS/MIC 2012; Anaheim, CA; United States; 29 October 2012 through 3 November 2012; Category number CFP12NSS-ART; Code 98448. Article number 6551194, Pages 693-696 DOI: 10.1109/NSSMIC.2012.6551194

[10] D. Mengoni et al., "Digital pulse-shape analysis with a TRACE early silicon prototype", Nucl. Instrum. And Meth., NIMA-D-14-00197R1. Volume 764, 11 November 2014, Pages 241-246 DOI: 10.1016/j.nima.2014.07.054

[11]Pullia, A., Capra, S., Frontini, E., "Design of an integrated low-noise ultra-fast charge-sensitive micro-probe for semiconductor detectors", 2012 IEEE Nuclear Science Symposium and Medical Imaging Conference Record, NSS/MIC 2012; Anaheim, CA; United States; 29 October 2012 through 3 November 2012; Category numberCFP12NSSART; Code 98448, Article number 6551954, Pages 4179-4183, DOI: 10.1109/NSSMIC.2012.6551954

[12] Aliaga, R.J., Herrero-Bosch, V., Capra, S., (...), Gadea, A., Mengoni, D., "Conceptual design of the TRACE detector readout using a compact, dead time-less analog memory ASIC", Published in Nuclear Instruments and Methods in Physics Research, Section A: Accelerators, Spectrometers, Detectors and Associated Equipment, Volume 800, 11 November 2015, Pages 34-39 DOI: 10.1016/j.nima.2015.07.067

[13] A. Pullia, D. Barrientos, D. Bazzacco, M. Bellato, D. Bortolato, R. Isocrate, "A 12-channel 14/16-bit 100/125-MS/s digitizer with 24-Gb/s optical output for AGATA/GALILEO", 2012 IEEE Nuclear Science Symposium and Medical Imaging Conf. Rec., ISBN 978-1-4673-2028-3, pp. 819-823, Anaheim, CA, Oct. 27 2012-Nov. 3 2012, DOI: 10.1109/NSSMIC.2012.6551218

[14]D. Barrientos et al, "Multiple register synchronization with a high-speed serial link using the aurora protocol", IEEE Trans. Nucl. Sci., vol. 60, no. 5 , pp. 3521-3525, 2013, Article number 6583338, DOI: 10.1109/TNS.2013.2273369

[15]D. Barrientos et al., "Performance of the Fully Digital FPGA-Based Front-End Electronics for the GALILEO Array", IEEE Trans. Nucl. Sci., in press, 2015, DOI: 10.1109/TNS.2015.2480243 